\title{
Dielectric Susceptibility of Liquid Water: Microscopic Insights from Coherent and Incoherent Neutron Scattering
}

\author{
A. Arbe, ${ }^{1}$ P. Malo de Molina, ${ }^{1}$ F. Alvarez,${ }^{1,2}$ B. Frick,${ }^{3}$ and J. Colmenero ${ }^{1,2,4, *}$ \\ ${ }^{1}$ Centro de Física de Materiales (CFM) (CSIC-UPV/EHU)—Materials Physics Center (MPC), \\ Paseo Manuel de Lardizabal 5, 20018 San Sebastián, Spain \\ ${ }^{2}$ Departamento de Física de Materiales (UPV/EHU), Apartado 1072, 20080 San Sebastián, Spain \\ ${ }^{3}$ Institut Laue-Langevin, 71 Avenue des Martyrs, CS 20156, F-38042 Grenoble Cedex 9, France \\ ${ }^{4}$ Donostia International Physics Center, Paseo Manuel de Lardizabal 4, 20018 San Sebastián, Spain
}

(Received 16 April 2016; published 24 October 2016)

\begin{abstract}
The analysis of neutron scattering results on $\mathrm{H}$ dynamics $\left(\mathrm{H}_{2} \mathrm{O}\right)$ and the dynamic structure factor $\left(\mathrm{D}_{2} \mathrm{O}\right)$ around the intermolecular peak and at intermediate length scales in terms of the susceptibilities reveals three processes (diffusive, local relaxational and vibrational) at frequencies below $3 \mathrm{THz}$, to which the contributions commonly invoked in dielectric studies can be directly mapped. We achieve a unified description of the results from both techniques, clarifying the nature of the molecular motions involved in the dielectric spectra and their impact on the structural relaxation.
\end{abstract}

DOI: 10.1103/PhysRevLett.117.185501

Water dynamics has paramount importance in many areas of research and industrial applications. One of the main techniques used from the early times to investigate water dynamics is dielectric spectroscopy (DS) [1]. Thanks to the development of the terahertz $(\mathrm{THz})$ techniques [2-4] it was recently possible to fill the gap between dipolar relaxation and intermolecular stretching vibrations at $\approx 5 \mathrm{THz}$, and to have a full picture of the dielectric permittivity $\varepsilon^{\star}(\nu)$ of liquid water in a broad frequency range. This is displayed in Fig. 1(a), which includes data from different sources at $298 \mathrm{~K}[2,5]$. The main contribution to the imaginary part of $\varepsilon^{\star}(\nu), \varepsilon^{\prime \prime}(\nu)$, is the well-known Debye peak centered at $\nu_{\max } \approx 20 \mathrm{GHz}$, which corresponds to a single exponential decay of the sample polarization with a characteristic time $\tau_{D}=\left(2 \pi \nu_{\max }\right)^{-1} \approx 8.3$ ps. This peak-which is also present in other hydrogen-bond (HB) liquids at different frequencies-is associated with the collective relaxation of the dipole moment $\vec{M}(t)=$ $\sum_{i} \vec{\mu}_{i}(t)$, with $\vec{\mu}_{i}(t)$ the dipole of the $i$ th water molecule. Figure 1(a) also shows that this peak is strongly suppressed in the susceptibility $\chi^{\star}(\nu)$ measured by light scattering (LS) $[6,7]$. In addition to this main contribution, other low amplitude processes have recently been invoked to describe the high-frequency part of the spectrum $[2,5,6,8]$. In Fig. 1(a) we have reproduced the most recently proposed description [5], which includes two additional Debye-like processes with characteristic times $\tau_{2} \approx 1$ and $\tau_{3} \approx 0.18 \mathrm{ps}$. Despite the evident need for two additional contributions to the main Debye peak to properly describe $\varepsilon^{\prime \prime}(\nu)$, the situation is still confused. The values of $\tau_{2}$ and $\tau_{3}$ are rather scattered (see Ref. [5] for a recent compilation) and strongly depend on the model function used for process 3 [5]. Moreover, the interpretation of the molecular motions involved in the different processes is very unclear, mainly because DS is a "macroscopic" technique, which follows the total dipole moment $\vec{M}(t)$ without spatial resolution.

The relevant frequency range for the dielectric response of water can also be covered by neutron scattering (NS), a technique delivering microscopic information with space and time resolution. NS has advantages for identifying processes at the $\mathrm{THz}$ range, avoiding interferences from the peak at $5 \mathrm{THz}$, which is barely visible by NS. More importantly, by measuring $\mathrm{D}_{2} \mathrm{O}$ samples NS reveals the dynamic structure factor $S(Q, \nu)$, with $Q$ the wave vector, i.e., it allows following the actual structural relaxation [9]. However, apart from a few exceptions [10,11], most of the NS studies of water dynamics, from the paper of Teixeira et al. [12], have been focused on incoherent scattering from protonated samples (see Ref. [13] for a critical discussion of the works carried out). Although the synergetic combination of NS and DS has proven to be a powerful tool in different but likely related problems as, for instance, polymer melt dynamics [14], this methodology has never been explored for water dynamics. With these ideas in mind, we have considered incoherent and coherent NS in a wide $Q$ range covering the first maximum of the static structure factor $S(Q)\left(Q_{\max } \approx 2 \AA^{-1}\right)$ and the so-called intermediate $Q$ range $\left(0.3 \AA^{-1} \lesssim Q \lesssim Q_{\max }\right)$. The NS data were analyzed in terms of the corresponding susceptibility $\chi_{Q}^{\star}(\nu)$. Its imaginary part can be calculated as $\chi_{Q}^{\prime \prime}(\nu) \propto$ $S(Q,-\nu) / n(\nu)$ from the scattering function corresponding to 'system energy loss' with $n(\nu)=\left(e^{h \nu / k T}-1\right)^{-1}$ the Bose occupation factor ( $k$ : Boltzmann constant) (see the Supplemental Material [15]). This less conventional analysis of NS data allows distinguishing better the different processes involved in $S(Q, \nu)$ and a more direct comparison with spectroscopy data. 


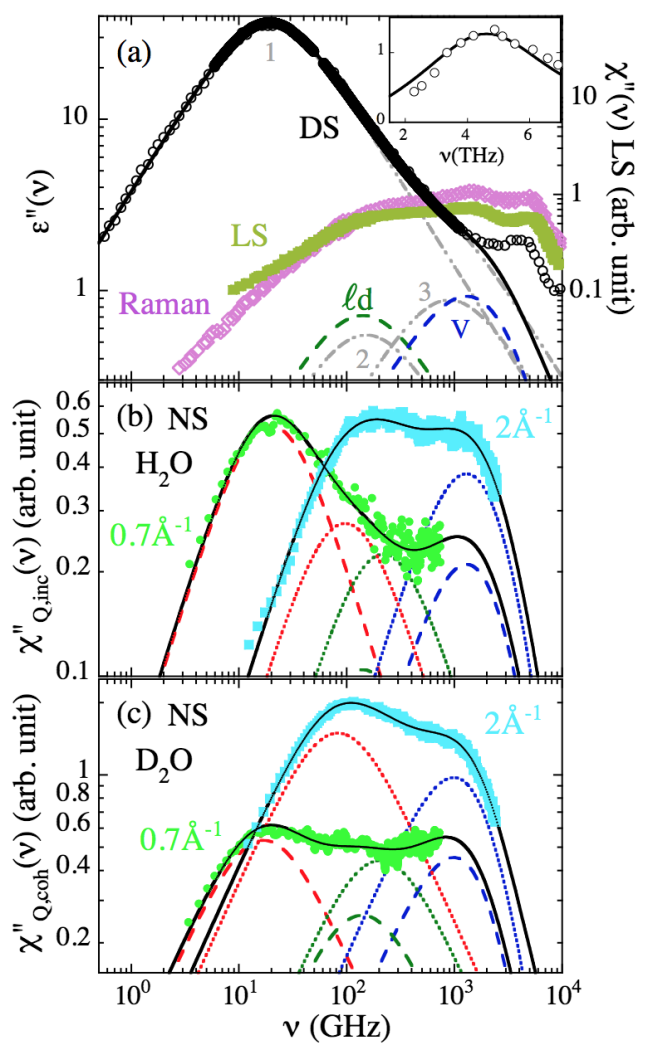

FIG. 1. Imaginary part of water susceptibility at 298 K. (a) DS (closed [5] and open [2] circles), Raman [6] (diamonds) and LS [7] (squares; $T=293 \mathrm{~K}$ ) results. Lines are fitting curves of DS results from Ref. [5] and respective components: dashed-dotted lines correspond to the fit proposed in Ref. [5] with processes 1 (main Debye), 2, and 3; solid line to the fit obtained in this work with process $d$ for $Q^{\star}=0.7 \AA^{-1}$ and processes $\ell d$ and $V$ (dashed lines). Inset: difference between the DS results and our fit, and model resonance given in Ref. [3] (line). (b) Incoherent NS results. (c) Coherent NS results. In (b) and (c), black solid lines are fits with the three components (red, diffusive; green, effective local; blue, vibrational) to the data at $Q=0.7 \AA^{-1}$ (circles, dashed lines) and $2.0 \AA^{-1} \approx Q_{\max }$ (squares, dotted lines).

The NS experiments were carried out at $298 \mathrm{~K}$ on $\mathrm{H}_{2} \mathrm{O}$ and $\mathrm{D}_{2} \mathrm{O}$ samples for incoherent and coherent scattering, respectively, by the time-of-flight instrument IN5 [16] at the ILL. Diffraction measurements with polarization analysis [17] were also performed at $298 \mathrm{~K}$ on the D7 (ILL) instrument [18]. See the Supplemental Material for experimental details [15].

The $\chi_{Q}^{\prime \prime}(\nu)$ obtained for the incoherent and coherent case and two $Q$ values are shown in Figs. 1(b) and 1(c), respectively. A first qualitative inspection of $\chi_{Q}^{\prime \prime}(\nu)$ (see Figs. S2 and S3 for other $Q$ values) suggests the presence of three different processes. The one dominating at low frequencies shows dispersion in $Q$, indicating diffusive behavior. In the other extreme of the spectra, the relevant process shows a $Q$-independent and rather high characteristic frequency $(\approx \mathrm{THz})$ suggesting an inelastic vibrational origin. We note that the vibrational density of states of liquid water measured by NS has a low-frequency main peak centered at $\approx 2 \mathrm{THz}$ [19], which was identified with bending fluctuations of O-O-O units in the water-molecule network. The presence of a third intermediate process is more evident in the low- $Q$ coherent data. This process seems to be also roughly $Q$ independent, suggesting some kind of localized process. Based on these qualitative arguments, to fit the data we have first considered the addition of a vibrational and a relaxational contribution. In the time domain this general expression reads

$$
F(Q, t)=[1-C(Q)] F_{V}(Q, t)+C(Q) F_{R}(Q, t) .
$$

$F(Q, t)$ represents either the intermediate incoherent scattering function for $\mathrm{H}$ nuclei $S_{\text {inc, } \mathrm{H}}(Q, t)$ or the normalized dynamic structure factor $S(Q, t) / S(Q)$-functions related through Fourier transformation with those measured on the protonated and deuterated samples, respectively. For the relaxational contribution we have assumed the convolution of two independent processes: a diffusive contribution $F_{d}(Q, t)$ and a local-restricted in space-contribution $F_{\ell}(Q, t)$. In the time domain this convolution reduces to a simple product: $F_{R}(Q, t)=F_{d}(Q, t) F_{\ell}(Q, t)$. We note that a similar procedure was previously used to describe both NS [20] and DS data [21] of a qualitatively similar problem: the merging of the $\alpha$ relaxation and the local $\beta$ process in glass-forming polymers. The same scheme has also been applied to describe MD-simulation data of water $[13,22]$. Here we assume that $F_{d}(Q, t)=e^{-t / \tau_{d}}$ with $\tau_{d}(Q)$ a diffusive time. For $F_{\ell}(Q, t)$ we take $F_{\ell}(Q, t)=$ $A(Q)+[1-A(Q)] e^{-t / \tau_{\ell}}$, where $\tau_{\ell}$ is a $Q$-independent relaxation time. Then, the relaxation contribution becomes $F_{R}(Q, t)=[1-A(Q)] e^{-t / \tau_{\ell d}}+A(Q) e^{-t / \tau_{d}}$, where the first term-with the effective local time $\tau_{\ell d} \equiv\left(1 / \tau_{\ell}+1 / \tau_{d}\right)^{-1}$ means the local process modified by the presence of the diffusive process and $A(Q)$ the relative amplitude of the pure diffusive process. According to Eq. (1) with this $F_{R}(Q, t), \chi_{Q}^{\prime \prime}(\nu)$ has three contributions: $\chi_{Q}^{d \prime \prime}(\nu)=C(Q) \times$ $A(Q) 2 \pi \tau_{d} \nu /\left[1+\left(2 \pi \tau_{d} \nu\right)^{2}\right], \chi_{Q}^{\ell d \prime \prime}(\nu)=C(Q)[1-A(Q)] \times$ $2 \pi \tau_{\ell d} \nu /\left[1+\left(2 \pi \tau_{\ell d} \nu\right)^{2}\right]$, and the vibrational contribution $\chi_{Q}^{V \prime \prime}(\nu)$. To represent the latter, we have assumed a resonance term as $\chi_{Q}^{V \prime \prime}(\nu)=[1-C(Q)] \nu_{0} \nu\left(k_{0} / 2 \pi\right) /\left[\left(\nu_{0}^{2}-\nu^{2}\right)^{2}+\right.$ $\left.\left(\nu k_{0} / 2 \pi\right)^{2}\right]$. Here, $\nu_{0}$ is the frequency and $k_{0}$ is the damping coefficient of the damped resonance. For incoherent scattering, $\chi_{\mathrm{Q}, \text { inc }}^{\prime \prime}(\nu)=\sum \chi_{\mathrm{Q}, \text { inc }}^{\alpha}{ }^{\prime \prime}(\nu)$, and for coherent scattering, $\chi_{\mathrm{Q}, \mathrm{coh}}^{\prime \prime}(\nu)=S(Q) \sum \chi_{\mathrm{Q}, \mathrm{coh}}^{\alpha}{ }^{\prime \prime}(\nu)$, with $\alpha=d, \ell d, V$.

The fitting curves of $\chi_{Q}^{\prime \prime}(\nu)$ are shown in Figs. 1(b) and 1(c) (and for more $Q$ values in Figs. S2 and S3). They nicely describe the experimental results. The values obtained for the vibrational parameters are $\nu_{0}^{\mathrm{H}_{2} \mathrm{O}}=2.64$ and $k_{0}^{\mathrm{H}_{2} \mathrm{O}}=38.7 \mathrm{THz} ; \nu_{0}^{\mathrm{H}_{2} \mathrm{O}}=1.75$ and $k_{0}^{\mathrm{H}_{2} \mathrm{O}}=22.5 \mathrm{THz}$. They translate in a characteristic frequency $\nu_{\max }^{V}$, 
corresponding to the maximum of $\chi_{Q}^{V \prime \prime}(\nu)$, of $\nu_{\max }^{V}=$ $1.3 \mathrm{THz}$ for $\mathrm{H}_{2} \mathrm{O}$ and $\nu_{\max }^{V}=0.975 \mathrm{THz}$ for $\mathrm{D}_{2} \mathrm{O}$. Their ratio is, as expected from the isotopic effect, about $\sqrt{2}$. On the other hand, the $\tau_{\ell}$ values of $\mathrm{D}_{2} \mathrm{O}$ and $\mathrm{H}_{2} \mathrm{O}$ samples scattered around $1.3 \mathrm{ps}$. Then we fixed $\tau_{\ell} \equiv 1.3 \mathrm{ps}$ for both cases. The rest of the parameters involved $[C(Q), A(Q)$, and $\tau_{d}(Q)$ ] depend on $Q$ and are presented in Fig. 2. This figure also includes for comparison the ratio between coherent and incoherent differential cross sections of the $\mathrm{D}_{2} \mathrm{O}$ sample as a measure of $S(Q)$. Panel (a) shows $C(Q)$ and $A(Q)$. For the incoherent case a Debye-Waller factor (DWF) like approach $\left[C(Q), A(Q) \propto \exp \left(-\left\langle u^{2}\right\rangle Q^{2} / 3\right)\right]$ delivers mean-squared-amplitudes (MSA) $\left\langle u_{V}^{2}\right\rangle=0.22 \AA^{2}$ (vibration) and $\left\langle u_{\ell}^{2}\right\rangle=0.28 \AA^{2}$ (local process). Within this approximation, $\left\langle u_{V}^{2}\right\rangle+\left\langle u_{\ell}^{2}\right\rangle$ would mean the MSA of the total nondiffusive process. We note that, although $A(Q)$ may be regarded as the EISF of the local process [13], the available data do not allow going beyond an effective DWF interpretation. The coherent amplitudes display a more complex $Q$ dependence involving some modulation
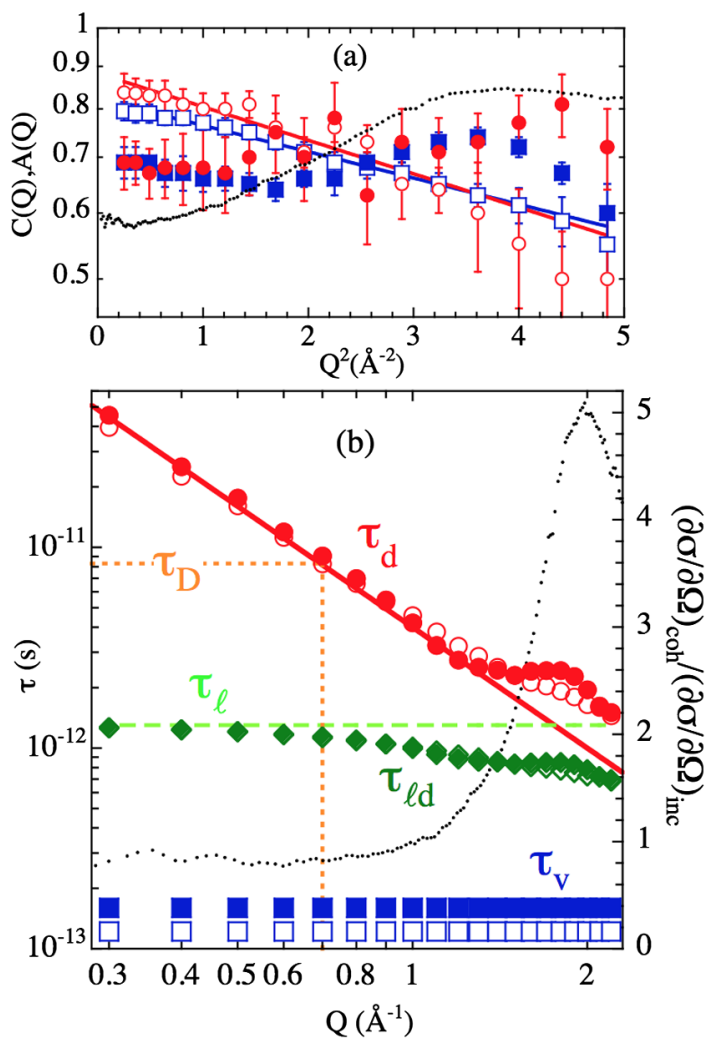

FIG. 2. $Q$ dependence of the parameters characterizing $\chi_{\mathrm{Q}, \text { inc }}^{\prime \prime}$ (open symbols) and $\chi_{\mathrm{Q}, \text { coh }}^{\prime \prime}$ (closed symbols). (a) Amplitudes $C(Q)$ (squares) and $A(Q)$ (circles). Lines: DWF-like fits. (b): Characteristic times corresponding to the diffusive (circles), vibrational (squares), effective local (diamonds), and local (dashed line) processes. Solid line: fit of the low- $Q$ incoherent times to $\tau_{d} \propto Q^{-2}$. D7 results for $\mathrm{D}_{2} \mathrm{O}$ are shown for comparison (dots) in both panels [in arb. units in (a)]. with $S(Q)$. The local component is highly visible in the intermediate $Q$ regime [1-A( $\left.\left.Q \approx 1 \AA^{-1}\right) \approx 0.32\right]$ as it was predicted in the above mentioned scenario for the $\alpha \beta$ merging [20,21]. Figure 2(b) shows $\tau_{d}(Q), \tau_{\ell}$, and the vibrational time $\tau_{V}=\left(2 \pi \nu_{\max }^{V}\right)^{-1}$. This time is $\tau_{V}=$ 0.16 ps for $\mathrm{D}_{2} \mathrm{O}$ and $\tau_{V}=0.12$ ps for $\mathrm{H}_{2} \mathrm{O}$. In the low$Q$ range where $S(Q)$ is almost flat, $\tau_{d}(Q)$ obtained either from coherent or from incoherent scattering is the same, within the uncertainties. $\tau_{d}(Q)$ from incoherent scattering deviates from the purely diffusive behavior at high $Q$ values, where it approaches $\tau_{\ell}$. The collective $\tau_{d}(Q)$ exhibits-as expected-some kind of "deGennes narrowing" [23] in the vicinity of $Q_{\max }$. We note that in the glass-forming community the $\alpha$ relaxation is identified with the structural relaxation leading to the decay of $S(Q, t)$ at the intermolecular distances, i.e., at $Q_{\max }$. Therefore, $\tau_{\alpha}$ is the average relaxation time of the relaxation contribution to $S\left(Q_{\max }, t\right) / S\left(Q_{\max }\right)$. According to $F_{R}(Q, t), \tau_{\alpha}=\left[1-A\left(Q_{\max }\right)\right] \tau_{\ell d}\left(Q_{\max }\right)+A\left(Q_{\max }\right) \tau_{d}\left(Q_{\max }\right)$, where all the parameters correspond to coherent scattering. Taking $A\left(Q_{\max }\right)=0.77$ [see Fig. 2(a)] $\tau_{\alpha}=0.23 \tau_{\ell d}\left(Q_{\max }\right)+0.77 \tau_{d}\left(Q_{\max }\right) \approx 1.7$ ps. Then, $\tau_{\alpha}$ has contributions from both local (through $\tau_{\ell d}$ ) and diffusive processes, although it seems to be dominated by $\tau_{d}$ at least at $298 \mathrm{~K}$.

Comparing now the time scales identified by NS with those reported in the DS studies [5], we observe that (i) $\tau_{D}=8.37 \mathrm{ps}$ coincides with $\tau_{d}\left(Q=Q^{\star} \approx 0.7 \AA^{-1}\right)$; (ii) $\tau_{\ell}$ and $\tau_{V}$ are in the range usually reported for the additional high-frequency processes of $\varepsilon^{\prime \prime}(\nu)$. Then, we have tried to fit the DS spectrum by the same model used for the neutron susceptibility at $Q^{\star} \approx 0.7 \AA^{-1}$. We have fixed the two time scales involved $\left[\tau_{D}=\tau_{d}\left(Q^{\star}=0.7 \AA^{-1}\right)\right.$; $\left.\tau_{\ell}=1.3 \mathrm{ps}\right]$ and the vibrational contribution of $\mathrm{H}_{2} \mathrm{O}$. Thereby, the only free fitting parameters were the two amplitude factors $C$ and $A$. As in Ref. [5], the fitting was restricted to $\nu \leq 1 \mathrm{THz}$ to minimize the influence of the peak at $\approx 5 \mathrm{THz}$ not included in the model. Figure 1(a) shows the perfect description of the DS spectrum in the considered frequency range. Moreover, the subtraction of the fitting curve from the experimental data at $\nu>2 \mathrm{THz}$ (shown in the inset) can be well described by the expression and the parameter values given by Yada et al. [3] for the intermolecular stretching vibrational peak. These are remarkable results taking into account that 4 out of 6 fitting parameters were already fixed. The values obtained, $A=0.98$ and $C=0.98$, translate into relative amplitudes to the DS spectrum (96.04\% for the Debye peak, $1.96 \%$ for the effective local process, and $2 \%$ for the vibrational contribution) that are in the range of those previously reported [5]. Figure 1(a) also shows the three contributions of our model. Our effective local process $(\ell d)$ and our vibrational contribution almost coincide with the processes called 2 and 3 in Ref. [5]. This agreement allows the univocal identification of these DS contributions; in particular, the vibrational nature of process 3 , due to 
intermolecular fluctuations of the HB network-mainly O-O-O bending modes [19,24,25]. As expected [19], the relative contribution of this process for NS is larger than for DS. On the other hand, the above introduced $Q^{\star}-$ which means a link between molecular diffusion and dipolar relaxation-can be expressed (see SM [26]) as $Q^{\star}=\left[D \tau_{D}\right]^{-1 / 2}$, where $D$ is the diffusion coefficient. With the values of $D(T)$ [13] and $\tau_{D}(T)$ [8,30], $Q^{\star} \approx$ $0.7 \AA^{-1}$ independent of temperature in the range $270 \mathrm{~K}-$ $330 \mathrm{~K}$ (see SM [26]). With some approximations $Q^{\star}$ can also be expressed as $Q^{\star} \approx\left[(2 / 3) a^{2} G_{K} / J_{K}\right]^{-1 / 2}$, i.e., in terms of a "single-molecule" magnitude - the effective radius, $a$ - and a factor, $G_{K} / J_{K}$, measuring the strength of many-body-effects on dipolar relaxation $\left(G_{K}\right.$ is the Kirkwood static parameter and $J_{K}$ the Kirkwood dynamical coupling [31]). If we use $Q^{\star}=$ $0.7 \AA^{-1}$ and reported values [32,33] for $a(\sim 1.3-1.44 \AA)$ the above expression delivers $G_{K} / J_{K} \sim 1.5-2$, in the range usually reported $[34,35]$.

To get information about the atomic displacements at the time scales of the different processes, we have calculated the $\mathrm{H}$ mean squared displacement (MSD) $\left\langle r_{\mathrm{H}}^{2}(t)\right\rangle$ from $S_{\text {inc,H }}(Q, t)$, by assuming the Gaussian approximation: $\left\langle r_{\mathrm{H}}^{2}(t)\right\rangle=-6 \ln \left[S_{\mathrm{inc}, \mathrm{H}}(Q, t)\right] / Q^{2}$. The results obtained
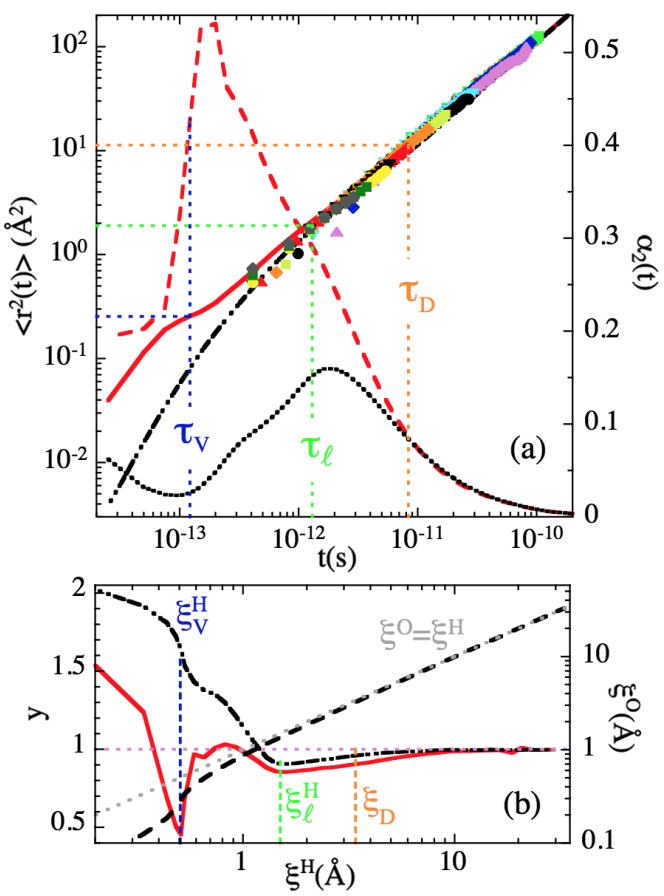

FIG. 3. (a) MSD experimentally obtained for $\mathrm{H}$ atoms (different symbols for different $Q$ values in the range $0.19 \leq Q \leq 2.0 \AA^{-1}$ ) and calculated from the simulations for $\mathrm{H}$ (solid line) and $\mathrm{O}$ atoms (dashed-dotted line). The computed $\alpha_{2}(t)$ are shown as dashed ( $\mathrm{H}$ atoms) and dotted (O atoms) lines. (b) Effective power exponent $y$ for $\mathrm{H}$ (solid line) and $\mathrm{O}$ atoms (dashed-dotted line) and mean displacement of $\mathrm{O}$ atoms $\xi^{\mathrm{O}}$ (dashed line) as functions of the mean displacement of $\mathrm{H}$ atoms $\xi^{\mathrm{H}}$. Gray dotted line: $\xi^{\mathrm{O}}=\xi^{\mathrm{H}}$-law. from different $Q$ values are shown in Fig. 3(a). Within the uncertainties, they lead to the same $\left\langle r_{\mathrm{H}}^{2}(t)\right\rangle$ for $t \geq 1 \mathrm{ps,}$ supporting the approximation in this range. This figure also includes the MSD and the non-Gaussian parameter $\alpha_{2}(t)=$ $3\left\langle r^{4}(t)\right\rangle /\left(5\left\langle r^{2}(t)\right\rangle^{2}\right)-1$ corresponding to $\mathrm{H}$ and $\mathrm{O}$ atoms calculated from the MD simulations carried out by us and described in the Supplemental Material [36]. In the time scale of the Debye peak, $\left\langle r^{2}\left(\tau_{D}=8.37 \mathrm{ps}\right)\right\rangle \approx 11.3 \AA^{2}$ for both atomic species. Thus, the collective dipolar relaxation can only take place when the atoms move in average large distances $\xi_{D}=\sqrt{\left\langle r^{2}\left(\tau_{D}\right)\right\rangle} \approx 3.4 \AA$, of the order of the intermolecular distance $2 \pi / Q_{\max }$. Large atomic displacements of $\approx 3.3 \AA$ were proposed in Refs. [38,39]—the socalled "tetrahedral displacement mechanism"-for explaining the Debye peak. Although our results prove the involvement of such large atomic displacements in the Debye peak, they cannot be identified with a characteristic hopping length as proposed for such a mechanism (see the Supplemental Material [40]).

The different dynamic regimes displayed in Fig. 3(a) are highlighted in Fig. 3(b), where we have represented the effective power exponent $y$ for $\mathrm{H}$ and $\mathrm{O}$ atoms, defined as $y=d\left[\log \left\langle r^{2}(t)\right\rangle\right] / d[\log t]$, as a function of the mean displacement of $\mathrm{H}$ atoms, $\xi^{\mathrm{H}}=\sqrt{\left\langle r_{\mathrm{H}}^{2}(t)\right\rangle}$. We note that $y=2$ corresponds to ballistic motion and $y=1$ to pure diffusion. A deep minimum in $y(t)$ would mean a spatial localization or delocalization process. Hydrogen atoms show a well-defined deep minimum at $\xi_{V}^{\mathrm{H}}=\sqrt{\left\langle r_{\mathrm{H}}^{2}\left(\tau_{V}\right)\right\rangle} \approx$ $0.5 \AA$. This first "cage" is vibrational and the decaging would likely involve HB breaking. In fact, the critical time separating "fluctuation and breaking" of the HB network has been estimated as $\tau_{c} \approx 0.3$ ps [44,45], which roughly corresponds to the end of this caging [see Fig. 3(a)]. Figure 3(b) also shows that this vibrational caging for $\mathrm{H}$ atoms is hardly reflected for $\mathrm{O}$ atoms. The second cage corresponds to mean displacements in the range of the local processes where $\xi_{\ell}^{\mathrm{H}} \approx \xi_{\ell}^{\mathrm{O}}$. This cage, which is visible for both $\mathrm{H}$ and $\mathrm{O}$ atoms, is less defined, likely due to the convolution of local and diffusive processes. Delocalization from this smooth cage leads to pure diffusive behavior, which for $\mathrm{O}$ atoms are established at $t \gtrsim \tau_{\mathrm{D}}\left(\xi^{\mathrm{O}} \gtrsim \xi_{\mathrm{D}}\right)$. In fact, the maximum of $\alpha_{2}^{\mathrm{O}}(t)$, usually marking the crossover to diffusive behavior [46], takes place at $\approx \tau_{\ell}$. Since the total reorientation of $\vec{M}(t)$ (collective Debye peak) requires large O displacements $(\approx 3.3 \AA)$, it is expected that the motions inside this cage $\left(\xi_{\ell}^{O} \approx 1.4 \AA\right)$ only contribute to hindered rotations of $\vec{M}(t)$, which translate into the low amplitude dipolar relaxation observed in this short-time-highfrequency range. In conclusion, we have achieved a unified description of NS and DS susceptibilities of liquid water, which (i) allows a microscopic interpretation of the different processes; (ii) identifies the molecular motions involved in the DS spectra; (iii) clarifies the nature of the actual 
structural relaxation time, $\tau_{\alpha}$; and (iv) provides a link between molecular diffusion and collective dipolar relaxation through $Q^{\star}$. This description also opens a new way of approaching dynamics of water under different conditions (supercooled, confined, etc.) and that of other H-bonded liquids.

We thank A. Wildes for his help at the D7 instrument at the ILL. Financial support by the Spanish Ministry 'Ministerio de Economia y Competitividad,'code: MAT2015-63704-P (MINECO/FEDER, UE) and by the Eusko Jaurlaritza (Basque Government), code: IT-654-13 is acknowledged.

*juan.colmenero@ehu.eus

[1] W. J. Ellison, K. Lamkaouchi, and J. M. Moreau, J. Mol. Liq. 68, 171 (1996).

[2] H. Yada, M. Nagai, and K. Tanaka, Chem. Phys. Lett. 464, 166 (2008).

[3] H. Yada, M. Nagai, and K. Tanaka, Chem. Phys. Lett. 473, 279 (2009).

[4] U. Heugen, G. Schwaab, E. Bründermann, M. Heyden, X. Yu, D. M. Leitner, and M. Havenith, Proc. Natl. Acad. Sci. U.S.A. 103, 12301 (2006).

[5] N. Q. Vinh, M. S. Sherwin, S. J. Allen, D. K. George, A. J. Rahmani, and K. W. Plaxco, J. Chem. Phys. 142, 164502 (2015).

[6] T. Fukasawa, T. Sato, J. Watanabe, Y. Hama, W. Kunz, and R. Buchner, Phys. Rev. Lett. 95, 197802 (2005).

[7] A. P. Sokolov, J. Hurst, and D. Quitmann, Phys. Rev. B 51, 12865 (1995).

[8] R. Buchner, J. Barthel, and J. Stauber, Chem. Phys. Lett. 306, 57 (1999).

[9] S. W. Lovesey, Theory of Neutron Scattering from Condensed Matter (Clarendon Press, Oxford, 1984).

[10] J. Teixeira, M. C. Bellissent-Funel, S. H. Chen, and B. Dorner, Phys. Rev. Lett. 54, 2681 (1985).

[11] J. Teixeira, A. Luzar, and S. Longeville, J. Phys. Condens. Matter 18, S2353 (2006).

[12] J. Teixeira, M.-C. Bellissent-Funel, S. H. Chen, and A. J. Dianoux, Phys. Rev. A 31, 1913 (1985).

[13] J. Qvist, H. Schober, and B. Halle, J. Chem. Phys. 134, 144508 (2011).

[14] A. Arbe, J. Colmenero, and D. Richter, in Broadband Dielectric Spectroscopy, edited by F. Kremer and A. Schönhals (Springer-Verlag, Berlin, Heidelberg, 2003), p. 685 .

[15] See Supplemental Material at http://link.aps.org/ supplemental/10.1103/PhysRevLett.117.185501, Sec. I, where the experiments, the accessed magnitudes in neutron scattering experiments, and the calculation of the susceptibility are described in more detail.

[16] J. Ollivier and J.-M. Zanotti, Collection SFN 10, 379 (2010).

[17] O. Schärpf, Physica (Amsterdam) 182B, 376 (1992).
[18] J. R. Stewart, P. P. Deen, K. H. Andersen, H. Schober, J.-F. Barthélémy, J. M. Hillier, A. P. Murani, T. Hayes, and B. Lindenau, J. Appl. Crystallogr. 42, 69 (2009).

[19] S. H. Chen and J. Teixeira, Adv. Chem. Phys. 64, 1 (1986).

[20] A. Arbe, U. Buchenau, L. Willner, D. Richter, B. Farago, and J. Colmenero, Phys. Rev. Lett. 76, 1872 (1996).

[21] A. Arbe, D. Richter, J. Colmenero, and B. Farago, Phys. Rev. E 54, 3853 (1996).

[22] J. Qvist, C. Mattea, E. P. Sunde, and B. Halle, J. Chem. Phys. 136, 204505 (2012).

[23] P. G. de Gennes, Physica (Amsterdam) 25, 825 (1959).

[24] I. Ohmine, J. Phys. Chem. 99, 6767 (1995).

[25] D. Russo, J. Teixeira, L. Kneller, J. R. D. Copley, J. Ollivier, S. Perticaroli, E. Pellegrini, and M. A. Gonzalez, J. Am. Chem. Soc. 133, 4882 (2011).

[26] See Supplemental Material at http://link.aps.org/ supplemental/10.1103/PhysRevLett.117.185501, Sec. II, where the derivation of the expression for $Q^{\star}$ is described in more detail. This section includes Refs. [27-29].

[27] T. Springer, in Quasielastic Neutron Scattering for the Investigation of Diffusive Motions in Solids and Liquids, Springer Tracts in Modern Physics, Vol. 64 (SpringerVerlag, Berlin Heidelberg New York, 1972).

[28] P. G. Wolynes and J. M. Deutch, J. Chem. Phys. 67, 733 (1977).

[29] T. D. Gierke, J. Chem. Phys. 65, 3873 (1976).

[30] U. Kaatze, Meas. Sci. Technol. 18, 967 (2007).

[31] D. Kivelson and P. Madden, Mol. Phys. 30, 1749 (1975).

[32] D. Braun, S. Boresh, and O. Steinhauser, J. Chem. Phys. 140, 064107 (2014).

[33] G. Sposito, J. Chem. Phys. 74, 6943 (1981).

[34] A. Chandra and B. Bagchi, J. Phys. Chem. 94, 3152 (1990).

[35] A. Volmari and Weingärtner, J. Mol. Liq. 98-99, 295 (2002).

[36] See Supplemental Material at http://link.aps.org/ supplemental/10.1103/PhysRevLett.117.185501, Sec. III, where the details of the molecular dynamics simulations are explained. This section includes Ref. [37].

[37] Discovery Studio 3.0.0.10321. Copyright (C) 2005-10, Accelrys Software Inc.

[38] N. Agmon, J. Phys. Chem. 100, 1072 (1996).

[39] V. I. Arkhipov and N. Agmon, Isr. J. Chem. 43, 363 (2003).

[40] See Supplemental Material at http://link.aps.org/ supplemental/10.1103/PhysRevLett.117.185501, Sec. IV, where the jump diffusion model is described. This section includes Refs. [27,41-43].

[41] K. S. Singwi and A. Sjölander, Phys. Rev. 119, 863 (1960).

[42] P. A. Egelstaff, An Introduction to the Liquid State (Oxford University Press, New York, 1992).

[43] A. Arbe, J. Colmenero, F. Alvarez, M. Monkenbusch, D. Richter, B. Farago, and B. Frick, Phys. Rev. E 67, 051802 (2003).

[44] J. J. Loparo, C. J. Fecko, J. D. Eaves, S. T. Roberts, and A. Tokmakoff, Phys. Rev. B 70, 180201 (2004).

[45] A. Luzar and D. Chandler, Phys. Rev. Lett. 76, 928 (1996).

[46] F. Sciortino, P. Gallo, P. Tartaglia, and S. H. Chen, Phys. Rev. E 54, 6331 (1996). 